Yozo Ohnishi · Toshihiro Tanaka • Kouichi Ozaki

Ryo Yamada · Hideyuki Suzuki • Yusuke Nakamura

\title{
A high-throughput SNP typing system for genome-wide association studies
}

\begin{abstract}
One of the most difficult issues to be solved in genome-wide association studies is to reduce the amount of genomic DNA required for genotyping. Currently available technologies require too large a quantity of genomic DNA to genotype with hundreds or thousands of singlenucleotide polymorphisms (SNPs). To overcome this problem, we combined the Invader assay with multiplex polymerase chain reaction (PCR), carried out in the presence of antibody to Taq polymerase, as well as using a novel 384-well card system that can reduce the required reaction volume. We amplified 100 genomic DNA fragments, each containing one SNP, in a single tube, and analyzed each SNP with the Invader assay. This procedure correctly genotyped 98 of the 100 SNP loci examined in PCR-amplified samples from ten individuals; the genotypes were confirmed by direct sequencing. The reproducibility and universality of the method were confirmed with two additional sets of 100 SNPs. Because we used 40ng of genomic DNA as a template for multiplex PCR, the amount needed to assay one SNP was only $0.4 \mathrm{ng}$; therefore, theoretically, more than 200,000 SNPs could be genotyped at once when $100 \mu \mathrm{g}$ of genomic DNA is available. Our results indicate the feasibility of undertaking genome-wide association studies using blood samples of only $5-10 \mathrm{ml}$.
\end{abstract}

Y. Ohnishi $\cdot$ T. Tanaka $\cdot$ K. Ozaki

Laboratory for Cardiovascular Diseases, SNP Research Center, The Institute of Physical and Chemical Research (RIKEN), Tokyo, Japan

R. Yamada

Laboratory for Rheumatic Diseases, SNP Research Center, The

Institute of Physical and Chemical Research (RIKEN), Tokyo, Japan

H. Suzuki $\cdot$ Y. Nakamura $(\square)$

Laboratory of Molecular Medicine, Human Genome Center,

Institute of Medical Science, The University of Tokyo, 4-6-1

Shirokanedai, Minato-ku, Tokyo 108-8639, Japan

Tel. +81-3-5449-5372; Fax +81-3-5449-5433

e-mail: yusuke@ims.u-tokyo.ac.jp

Y. Nakamura

Laboratory for Genotyping, SNP Research Center, The Institute of

Physical and Chemical Research (RIKEN), Tokyo, Japan
Key words Single nucleotide polymorphism (SNP) · Multiplex PCR · Invader assay · Genotyping · Association study · Taq antibody

\section{Introduction}

The pathogenesis of common diseases, such as hypertension and diabetes mellitus, is thought to be caused, in some part, by common genetic variations such as single-nucleotide polymorphisms (SNPs) (Lander and Schork 1994; Lander 1996; Risch and Merikangas 1996; Collins et al. 1997; Kruglyak 1999). Genome-wide association studies undertaken on the basis of large-scale genotyping of SNPs in thousands of DNA samples will become one of the most potent methods for identifying genes related to complex diseases.

The Invader (Third Wave Technologies, Madison, WI, USA) assay, a method for typing SNPs, combines structurespecific cleavage enzymes with universal fluorescent probes. This assay can be applied to high-throughput genotyping (Lyamichev et al. 1999; Mein et al. 2000), but, although it does not require polymerase chain reaction (PCR) amplification of genomic DNA (Ryan et al. 1999), it does require $100 \mathrm{ng}$ of genomic DNA to assay a single SNP. Therefore, only 1000 SNPs can be genotyped from $100 \mu \mathrm{g}$ of genomic DNA (equivalent to a 5- to 10-ml sample of whole blood). Hence, this assay is not applicable for SNP genotyping on the very large scale needed for genome-wide association studies, for which 50,000-300,000 SNPs should be typed in each individual's DNA.

To reduce the amount of DNA required to less than $1 \mathrm{ng}$ for the assay of a single SNP and to make genome-wide association studies feasible, we combined a multiplex PCR method with the Invader assay.

\section{Materials and methods}

Preparation of genomic DNA. Blood samples were collected from ten unrelated individuals after written informed 
consent was obtained from all participants. Genomic DNAs were prepared from white blood cells by standard methods.

Primer design. The sequences of the primers used in this study were the same as those developed for PCR amplification in our earlier SNP-discovery project. Information on that project, including the sequences of primers and PCR conditions, can be found on the World Wide Web at http://snp.u-tokyo.ac.jp.

Amplification of samples. Multiplexed amplification was performed with $40 \mathrm{ng}$ of genomic DNA. Each total reaction volume of $50 \mu \mathrm{l}$ contained $50 \mathrm{pmol}$ of each primer, 10 units of Ex-Taq DNA polymerase (TaKaRa Shuzo, Otsu, Japan), and $0.55 \mu \mathrm{g}$ of TaqStart (Clontech Laboratories, Palo Alto, CA, USA). Samples were amplified in the GeneAmp PCR system 9700 (Applied Biosystems, Foster City, CA). Initial denaturation was at $94^{\circ} \mathrm{C}$ for $2 \mathrm{~min}$, followed by 35 cycles of denaturation at $94^{\circ} \mathrm{C}$ for $15 \mathrm{~s}$, annealing at $60^{\circ} \mathrm{C}$ for $45 \mathrm{~s}$, and extension at $72^{\circ} \mathrm{C}$ for $3 \mathrm{~min}$.

Genotyping. The Invader assay combines structure-specific cleavage enzymes and a universal fluorescent resonance energy transfer (FRET) system. Allele-specific oligonucleotide pairs and invasive probes were designed and supplied by Third Wave Technologies. FRET probes were labeled with FAM or VIC that corresponded to alleles. Signal intensity was indicated as the ratio of FAM or VIC to ROX, an internal reference. Each total reaction volume of $10 \mu \mathrm{l}$ contained $0.5 \mu \mathrm{l}$ of signal buffer, $0.5 \mu \mathrm{l}$ of FRET probes, $0.5 \mu \mathrm{l}$ of structure-specific cleavage enzyme, $1 \mu \mathrm{l}$ of allelespecific probe mix, and $2 \mu$ l of PCR product diluted 1:10. Samples were incubated at $95^{\circ} \mathrm{C}$ for $5 \mathrm{~min}$, and then at $63^{\circ} \mathrm{C}$ for $15 \mathrm{~min}$, in an ABI7700 (Applied Biosystems). In this system, when correspondent alleles exist, sigmoid or quadratic signals are detected during incubation. On the other hand, when correspondent alleles do not exist, the signal is linear.

\section{Results}

First, we randomly selected 100 genomic DNA fragments in which we had discovered at least one SNP (the data are available through our SNP database, http://snp.ims.utokyo.ac.jp) and examined whether the DNA could be amplified sufficiently in a single tube for the Invader assay (Table 1). The 100 pairs of primers for DNA amplification were the same as those used for SNP screening. Simply mixing the 100 primer pairs with $40 \mathrm{ng}$ of genomic DNA failed to amplify some fragments sufficiently to be validated by the Invader assay, partly owing to dimerization of primer sequences (Edwards and Gibbs 1994). To overcome this problem, we added to the reaction solution anti-Taq polymerase antibody (TaqStart; Clontech Laboratories) (Kellogg et al. 1994), which is known to reduce dimerization and to increase the efficiency of PCR amplification of target amplicons. Some results are shown in Fig. 1.

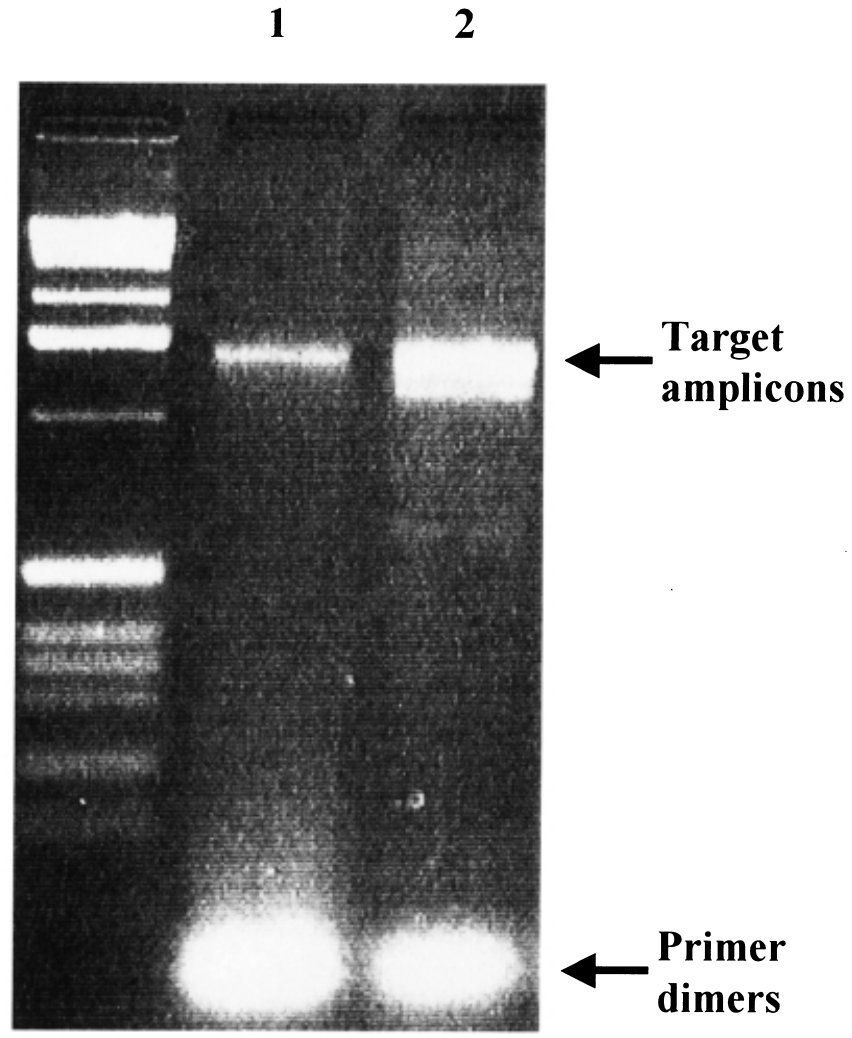

Taq-Ab - +

Fig. 1. Multiplex-polymerase chain reaction (PCR) products without (lane 1) and with (lane 2) anti-Taq polymerase antibody (Taq-Ab)

To evaluate the feasibility of amplifying multiple DNA fragments prior to assay by the Invader method, we attempted to genotype genomic DNAs from the ten unrelated individuals this way. After amplification was performed, using $40 \mathrm{ng}$ of genomic DNA as template, the Invader assay was performed with unique probes corresponding to each SNP allele. Figure 2a-c shows examples of real-time scanning of fluorescent signals, and indicates that $15-\mathrm{min}$ incubation at $63^{\circ} \mathrm{C}$ can discriminate sufficiently between alleles. To simplify the genotyping procedure, in the interests of developing a high-throughput automated scoring system, we investigated the possibility of measuring fluorescentsignal intensities in two dimensions at the end of the incubation. As shown in Fig. 2d, alleles could be discriminated at a glance. By examining 100 independent SNPs, each of which was present in one of 100 amplicons, we successfully obtained correct genotypes for 98 SNPs in the ten individuals. We examined the reproducibility of the method by repeating the experiment twice, independently, for the same 100 SNPs, and obtained identical results.

To confirm the accuracy of our method, we performed direct sequencing of 24 SNP loci in all ten samples of genomic DNA (a total of 240 fragments sequenced). The SNPs were randomly selected from among the 98 that had 
Table 1. Sequences of primers

Primer names

AC000353.27_20000214_5_FR AC000388.1_19970529_9_FR AC001643.1_19970529_3_FR AC002237.1_19970606_1_FR AC002319.1_19980203_3_FR AC002364.1_19981204_2_FR AC003005.1_19971022_1_FR AC003005.1_19971022_3_FR AC003689.1_19981121_2_FR AF066064.1_19980603_1_FR AF157101.1_1990624_1_FR AF196968.1_1991109_1_FR AJ009610.1_1990104_4_FR AJ011772.1_19981005_2_FR AJ011931.1_19981110_5_FR AJ229043.1_19990122_1_FR AL008633.1_19971029_1_FR AL008634.1_19981109_13_FR AL008634.1_19981109_14_FR AL008638.1_19981123_4_FR AL008730.1_19980204_2_FR AL008733.10_19991225_1_FR AL008734.10_19990610_1_FR AL021917.1_19980721_19_FR AL023279.1_19990305_3_FR AL049557.19_19990728_8_FR AL049569.13_19990803_29_FR AL049569.13 19990803 5 FR AL049569.13_19990803_12_FR AL049569.13_19990803_13_FR AL049575.7_19990701_2_FR AL049611.24_19991123_9_FR AL049612.11_19991123_4_FR AL049649.4_19990701_3_FR AL049650.8_19990706_9_FR AL049691.17_19991123 5_FR AL049694.9_19990730_1_FR AL049698.3 19991123 2 FR AL049758.11_19991214_7_FR AL049758.11_19991214_11_FR AL049759.10_19991212_11_FR AL049795.20_19991123 31_FR AL049829.2_19990617_16_FR AL049829.2 19990617_23 FR AL049843.18_20000215_4_FR AL096766.12_20000107_2_FR AP000168.1_19990702_5_FR AP000171.1_19990702 8 FR AP000347.1_19990616_10_FR AC004224.1_19980226_8_FR AP000350.1_19990616_1_FR AP000350.1_19990616_4_FR AP000352.1_19990616_11_FR AP000353.1_19990616_16_FR AC004448.1_19980702_1_FR AP000493.1_19990928 9 FR AP000495.1_19990928_7_FR AP000500.1_19990928_16_FR AP000500.1_19990928_18_FR AP000500.1_19990928 3 FR AP000501.1_19990928_18_FR AP000501.1_19990928_10_FR AP001041.1_20000114_2_FR AP001054.1_20000114_8_FR AC004465.1_19980711_2_FR AC004551.1_19980711_12_FR D50561.1_19990210_1_FR NT_002717.1_19991111_7_FR U07563.1_19960131_4_FR X56832.1_19970625_2_FR

Forward primers

Reverse primers

CCAGCAGGACTTGGTGACA G CA G C CA C C CA C T C A G T C T T G GCTTGAGACTCACCCTCTGATG TCTGCCAAGCAGAAACCTAGAG C T T T C C G A C A A C G A G A G C G A GGCCGTGA GGGA A T G A T G TTCAGCATAGCTCCAGAAGGC TGGCTCACTAAATGCACTACCAC A CCACA GGCTCCA GGA A GTG CACTCCCA C CA C CA TCACT G CGTTGGAAGCCTGTACTCCTTAG G GCA CCTCTCCA G G A T T G T G CCCAAGGCCCACTGTGTTAC GA GCAT T GCCCTCCTCACT G GCC T C T A C C T T T A C C G T C C G CAGCTTCAGGCCAAATGTATG TCCACCCT GA T CA A G T CCA G AAATTAAGGCACAGGCAGTGAG TCTATGTGGGTAGGATCTCCAGAC CA GCA GCA A CA A CA A CCG TC GAAATGCCTCCCTGGAACA G TCCCTGA GCCCA G G T A A G T C CATCCTCGTCACTGACTAATAGCG GCCA G G GA CT GA A G C T G A A C CACTTTGAGCACTCTCAGGAGAAC GAGA A CGGGCTGAGGACAAG CCCTGAGTCTAGCTCAAATCTCTC CTGA G G G T C C C T T CA C C A A G CCACTGTCCTGGCTCA GA T G GTGACCTTCCTCTGTCCTATTACG A T C C A C T G G C C A T T C T G C T G A GGCAGACAA A TCGCCACTC TGTGGGCTGCTCTGA G G TA G GGGA A GA CCCA GCCA T A A TC C T G G G C C T G T G T C T T C A C T G GCAGCCCTCTGACTATATGAGTTG GATTAGCGTTTCTTTCAGCCATC GGCCAA A G GTTCCA G G A A G C C T C C T CA GT T TC T C CA GCG A CCAATCCA A G G GCTA G G T G GTTACAAACCTGACTTGTGGCTC GCCAAACAATCCCTCATGATAC TCCCAACTCATTTCAGCATCTC T T A A C T G G C C C T G TCT G G T G GGGCTTCTTCT G CA T G T G G AAACCTCACTGTCTGCTTCCTG T T T G GCC T T G T T T G C C T C T G GAGTGTGGTCCATAAACTTGGC GCTGTGTGACGTTAGGCCA G G G C C A T T C C C T T T A T T G C G GCTTA TTCCTGCA A G G CGTC A A CCCTGA GCCTGTCACCTG T G T G A C C T T C C T G GCTCT T C CAACTGTGAGTGACCGTGGAG C C T GCTCCCACCA T G A A G T C CATAAGCCGAGTGGTACAGAGC T G G C T T GA G G T T C T G G C T T C CCTATGCTCAGCCAAGGTCAG CTGTGATGGGCTGCA GA A T CACCCAGTGCAGCCTTATAGC A CCACGGA GTCTGGCATCAC T T T G T C C T T G G G C T T G G T A G CCCATCCCGTTAAAGCACTTAG GTGTGCTTTGTTTGGTTTGCATAG GGAGAGGAGACAAACAGAGATGC T T G G A T A T G T G T G T G G C A G C CAAACAGGTCACATTTGCTGAAG GGA GGCCTGACA GCCA TA T C TTCTTTCTGCCA TCAAGTTGC TGTGATCTTCCAATTCCTCCTG
GCAAGAAGCAGCCAGATCAAG A G G T C C T G G C T C T G C G T A A C G T C C C G A C T T G A A G G T C C A C G G C A C C T T G A G A G G A A T G C CACCTGGACTCT GCA T C CT G G G G T G T C T A G CA T G G T G C T G TTTGGACCCTTGTCCTAACAAC A CCTGGAGGTGA A GCGA G A T G C G T T T G C A C T G G T A G G C GCTCACGGA A C T C G A A A C G A G G A GA GCTCA C C C G A A G T G G A A GCCA G G G C A A G TCA T T G C C T G G T GCAA G T G TCA A GTGCCACAATTGATATGACCAG G C C A C C T C C C T G T C T T C A T C T C A C A C C T C C T C C T C C A T T G G C A T G G G T G C A C T G T T A C G T C C T C T G C T T T G C T C A G G C TCGAAACAGAAGATGTGGCTG CCCAAGTGTGGTAGGTTTACAATG C T C T G C C A A G C C C A T C T T G T G T TCCCTGA TCCTCA TCCA G TCA A C A G C G A A C T C C A C C T G AAAGCATCAGTGGGCAGAATC GCT T T GA GCA A G G C T TCCA G T G C CA A G A A A G G G T G A C T G CCT GCTCCTTGA GCTTGTCAC GCAACAGCCTGAATGTACACAG GAGGATGTCACGGTTCCAGTC T T T CA G CA G G G A C A G A G T C G GCTCA A G G A G A C T GGTGTC T G CA T G G G C T T C A G T A G A G C C C CA C C C T C C T T T G G T A T G GAGTTGGTGGGCACTAAGGTG GGCAAAGGTCTTGGTGTCAAC A GA A CGCA GCA A GGA A GCAC TCTGAATTCCCATTCTTCATGC CGATGCAGAGACTGTCCAGAG T G G GCA T C T G A A T G G A A G C CAGGTCCAGCAGTGATCCATAC G GCTA T G A G T T C C C GCTCA G A T G C T T C C T C T A C CA T G GCG T G T C T G C C T C C C T G A C T C T G G T GCACACA GA G G T G T A G G T GCT T C C C A C T G T T C T C A G C CA G G T G A G A T C G G C A CA C T C A A G GCCACA T T T GA GA A C G A C CACGTCTCTA GCCA G T C G A G A T A C T G G G T T C C A T C C G C CCACAGGCCGTCTAGCTTATG A A T GGAAGCCAAAGGCACAG T GA GCCCTGA A T G C G A G T A G A GCCTCACT GACA T GCCT T G AGTGAGGTATTGGAATCTGAGGC A T G G A C T C C T A C A C G A T G C G TCCAAAGGCCATAGTTTACCAAG TGTGACGGGTA A GGCA GA T G A G A A C C A C C T G G G C T G C T A C G G A GA GCCTCCA G T T CA A G C A C C T C C C T C T C T G C C T T C T G CGGTCAGAACAAAGAGAGTGGAAC CAGGGAGAGGTATACGATGGTG A G G A T G G G C T T C C C A C T CA G C T G G G A A T G T G C C A G C A A G TGGGTACCA GA A A C T GCT GC G T G A T G T C T G C CA T G G A T G C TGGCCCACACAGACTAATAAGC GCCATATGTGGA A CA A GCA GC GCT T T G C CA G G A G C C T A G T G TA T G GCA G G A A A GA A G C A 
Table 1. Continued

\begin{tabular}{|c|c|c|}
\hline Primer names & Forward primers & Reverse primers \\
\hline X74107.1_19970625_17_FR & TGCACCAGACAGGGTAGCTG & CCATCCAGCCAAGTCCTTGTAG \\
\hline X78901.1_19970612_1_FR & CCAGACGGCTTAGAGCACTG & CGA G A G CA TC C A C C A G A GT G \\
\hline X87344.1_19980924_41_FR & GGACGGATGAAGATGAACGC & A T C C C A GCA GCCCTCT T A GC \\
\hline X91863.1_19961120_2_FR & T T GCCTTGA A C T T GCTCTGC & CACACCTGCCCTTTA T T G G TC \\
\hline Y08378.1_19970804_3_FR & TCTCCA GCAGGTACA GGCAC & G C C T T C A T C T C T G C A A G C T C \\
\hline Y12852.1_19971110_2_FR & ССТСАТGTССТGGTGCATTG & AGCTGCGAAGAGAGCCAGAG \\
\hline Y16792.1_19981106_4_FR & GATCACAGGTCGTAGGCAGATG & ССАТСCAGACTGGGTCAGTG \\
\hline Z54246.1_19960313_1_FR & C T C C A T G T G G G A A T C G G T G & TCA A G GCACA T C A C A T G G T C \\
\hline Z93241.1̄__19990512__20_FR & CСCTGAAGCTAAACATCACCAG & TCT G T C C C T G T G T G A C C T G C \\
\hline Z93241.11_19990512_21_FR & TCATGGTGAAGGGAGTTCTGAG & CGGA TA T T T C A G T G GAGGC \\
\hline Z96074.4_19990621_3_FR & CA A G GTGGTCA G T C T G GCTC & TGGGAATCATTAAGAGATGCCTG \\
\hline Z97192.1_19980821_7_FR & TGTCCAGGAGCCTGCCTATC & GCCTCCAGT TCT GCCA ACTC \\
\hline Z97632.1_19990423_15_FR & TC C T C C & TGCAACTAAGGGTGAGTGTTCG \\
\hline Z97876.1_19971031_3_FR & CCATGATGAAAGGGAAATAGAGC & TGCTACGTAAGTGGTTGACGC \\
\hline Z98200.8_19991123_11_FR & TCTGTCCAGGTTTCTGTCATCTC & C C A A A C T T G G A G G T G G T G T C \\
\hline Z98257.1_19980311_8_FR & TGTCCAAGGCAGAGGAATCTC & CAAGAGAGCTACAGGGTGCTTG \\
\hline Z98258.1_19971009_3_FR & ACACCAAGCCTTTGA GGCAC & A GCA GCCCAAGA GCTCA G AC \\
\hline Z98259.1_19970804_3_FR & CGCAGGTGACTTCTTAGGAGAAC & GCA GCT G G A C G T T G T C A T T C \\
\hline AC000024.̄1_19981031_7_FR & CTGGGACTCTGGGACAGATG & TGGTGCAGCTGGACTCTATTG \\
\hline AC000029.17_19990924__21_FR & A ACCAGACCTCCAGCCACTC & ССТАCAAGGTTTGCCCTACACTAC \\
\hline AC000063.1_19961114_14_FR & ACCAGATGCATAGCAGTGGC & C C CA T C C C A GCA TCC T A G A \\
\hline AC002106.1_19970902_2_FR & CAATTCTCCTTTGCGA G G T G & T G C C A C T G T C G T T A A G C C T G \\
\hline AC002297.1_19970610_2_FR & A G T C C C T T C C C G G T C T T C T G & T GCT A T G A GCA G T G G T C A C G \\
\hline AC002297.1_19970610_3_FR & TCA T C C T T T C T G G G A T C C T G & C CA GCTGGCTGCATCTATTC \\
\hline AC002400.1_19991123_15_FR & A CCTTGGGTGCTGTA GGTGC & C C T C C A A G C T G G A G T T C C T C \\
\hline AC004556.1_19981211_2_FR & T GCCTTGGCCT A A G T G T G T G & C CA T GCA G T A GCT GA G GCT G \\
\hline AC002467.1_20000216_2_FR & ТСТСААТGTGCААА TGTGGC & ТСССТСССТССТTG TA TCCG \\
\hline AC005088.2_19990505_1_FR & GGAGGGAATAGCCAGCACTC & G A C C C T T T G A A G G T T G C T G C \\
\hline AC005137.1_19980930_1_FR & CTGGTCCCTGTCACCA G A A G & A G GTGGCTGTGA A A C T G GTG \\
\hline AC003026.1_19991123_10_FR & TGTTGATGCTGGGTGGA T TC & GCССТСТАTAATCAAACACGAGC \\
\hline
\end{tabular}

been genotyped successfully. The sequencing results were $100 \%$ identical to the genotyping data obtained by the Invader assays coupled with multiplex PCR amplification of the 100 genomic fragments. To confirm whether this method could be applied for any combination of SNPs, we evaluated it using two different sets of 100 SNPs selected from our database. The same experimental procedures again yielded high success rates; we obtained reliable signal intensities for 96 and 98 SNPs, respectively, in the two additional sets of 100 SNPs examined. We believe, therefore, that this method should be applicable to any combination of SNPs.

Furthermore, to achieve a higher throughput, we developed our original 384-well reaction card (Fig. 3a), in which only $1 \mu \mathrm{l}$ of reaction mixture is required for one SNP assay. After samples are spotted in the well, the card is sealed tightly with an overlaid cover plate that is welded on ultrasonically. As shown in Fig. 3b, to avoid reaction mixtures contaminating each other, grooves were prepared around the reaction wells. Multiple Invader assays, using this card, provided sufficiently high fluorescent signals.

\section{Discussion}

The greatest advantage of the system described here is the significant reduction in the amount of genomic DNA required for the genotyping of a single SNP - to $0.4 \mathrm{ng}$. Even if we were to carry out a genome-wide association study involving $100,000 \mathrm{SNPs}$, only $40 \mu \mathrm{g}$ of genomic DNA would

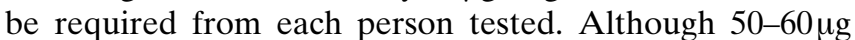
may be necessary for repeated experiments if genotyping fails for some SNPs, 5- to 10-ml samples of blood from any participant are likely to be sufficient. Our procedure also reduces the cost of the PCR to much less than $\$ 0.01$ per SNP. The improvements described here should make genome-wide SNP genotyping more realistic and should have a great impact on studies designed to identify genes that confer susceptibility to various common diseases.

Another advantage of our system is a reduction in the time required for the Invader assay. The flow-chart in Fig. 3c illustrates the mechanics of our high-throughput genotyping system. Although a multiplex PCR process is required prior to the Invader assay, the PCR time for all SNPs is considered to total only a few minutes, because 100 fragments are amplified together. And because of the high copy-number of each target DNA fragment amplified by the PCR, $20 \mathrm{~min}$ of incubation and $5 \mathrm{~min}$ for scanning and allele discrimination are sufficient; the original protocol took $4 \mathrm{~h}$. Furthermore, using our cards, we can incubate many samples simultaneously in a water bath at $63^{\circ} \mathrm{C}$, and, thus, there is no need to occupy scanning machines for incubation. By our estimation, we can carry out the genotyping of 300,000-400,000 SNPs in 1 day with our high-throughput system, and this is equivalent to genotyping about 100 million SNPs over the course of a year. 
Fig. 2a-d. Representative experimental results for an Invader assay after simultaneous PCR amplification of 100 genomic fragments. Signal intensities were recorded continuously during incubation in the ABI7700 (Applied Biosystems, Foster City, CA). Red curves indicate the intensity of the VIC signal divided by that of ROX, an internalcontrol fluorescent dye. Blue curves indicate relative FAM signals calculated in the same way. The respective fluorescent signals were detectable in homozygous individuals (a and $\mathbf{c}$ ) and both signals were detected in a heterozygous sample (b). Note that all signals reached a plateau within $15 \mathrm{~min}$. d Allelic discrimination of 30 samples (triplicate experiments with 10 samples). The horizontal axis shows the signal intensity of VIC/ROX; the vertical axis shows the signal intensity of FAM/ROX. The cluster of red spots indicates a strong $\mathrm{VIC/ROX}$ signal and a weak FAM/ROX signal; the samples in this cluster were judged to be homozygous for allele 1. Similarly, samples indicated by green spots are heterozygous, and samples yielding blue spots are homozygous for allele 2 a )

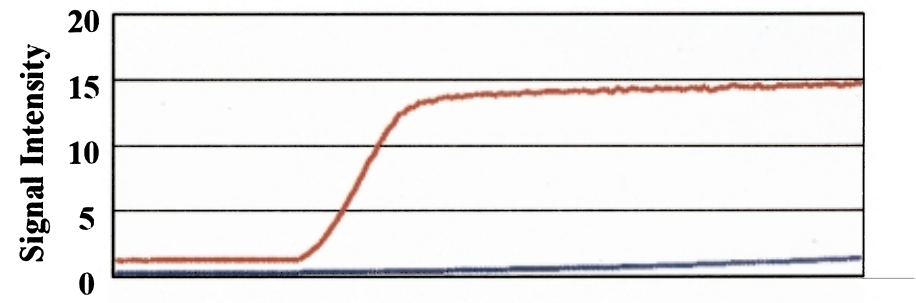

Homozygote of Allele Red

b )

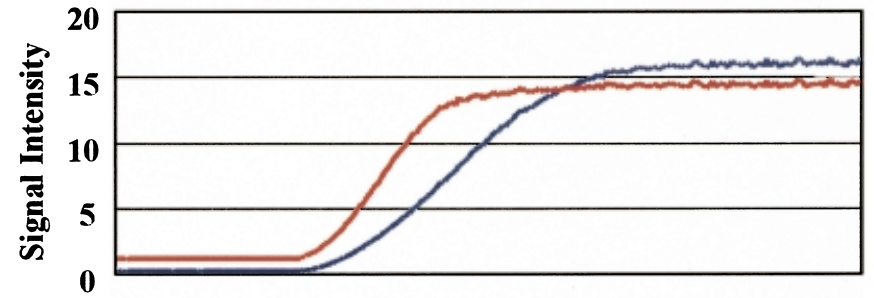

Heterozygote

c)

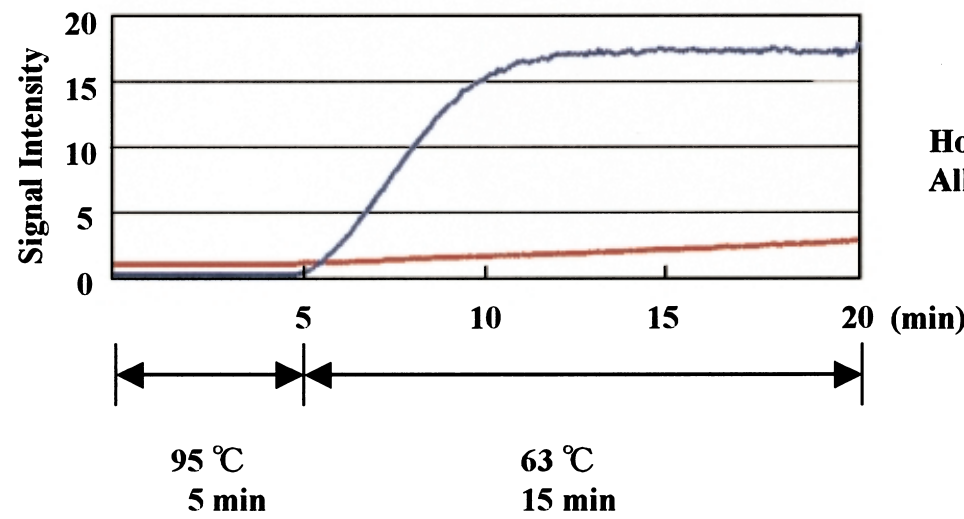

d)

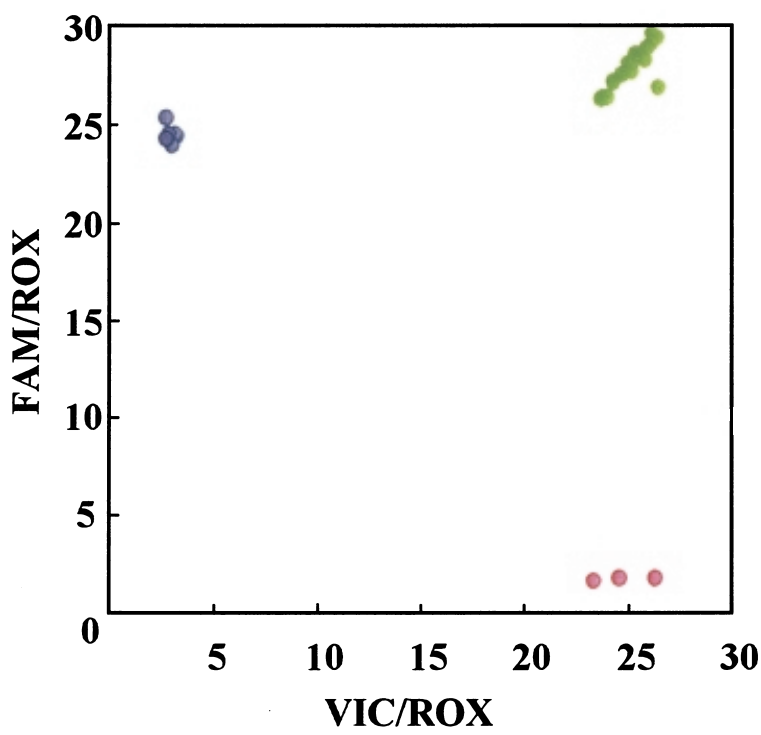

At present, more than $90 \%$ of the human genomic sequence has been determined, and the next decade will be an era of "functional genome analyses", including the identification of genes related to diseases with complex traits. Genome-wide association studies should play an important role in finding genes related to diseases that do not show
Mendelian inheritance. Because such studies will require the assay of hundreds of millions of SNPs, genotyping methods with very high throughput will be indispensable. Moreover, one of the most important issues in making genome-wide association studies feasible is the amount of genomic DNA required from each human participant: 
Fig. 3a-c. High-throughput genotyping system. a Overview of the 384-well reaction card. b Method of sealing up the samples. After the wells are filled with reaction solution, each well is covered with a plastic plate. At this point, some portion of the sample overflows from the well, to keep residual air bubbles to a minimum, and the overflow is trapped by the grooves surrounding the wells. c Experimental flow-chart for Invader assay procedure, after the simultaneous PCR amplification of 100 genomic fragments, also showing the calculated amount of DNA required in each step. SNP, Single-nucleotide polymorphism a)

b)
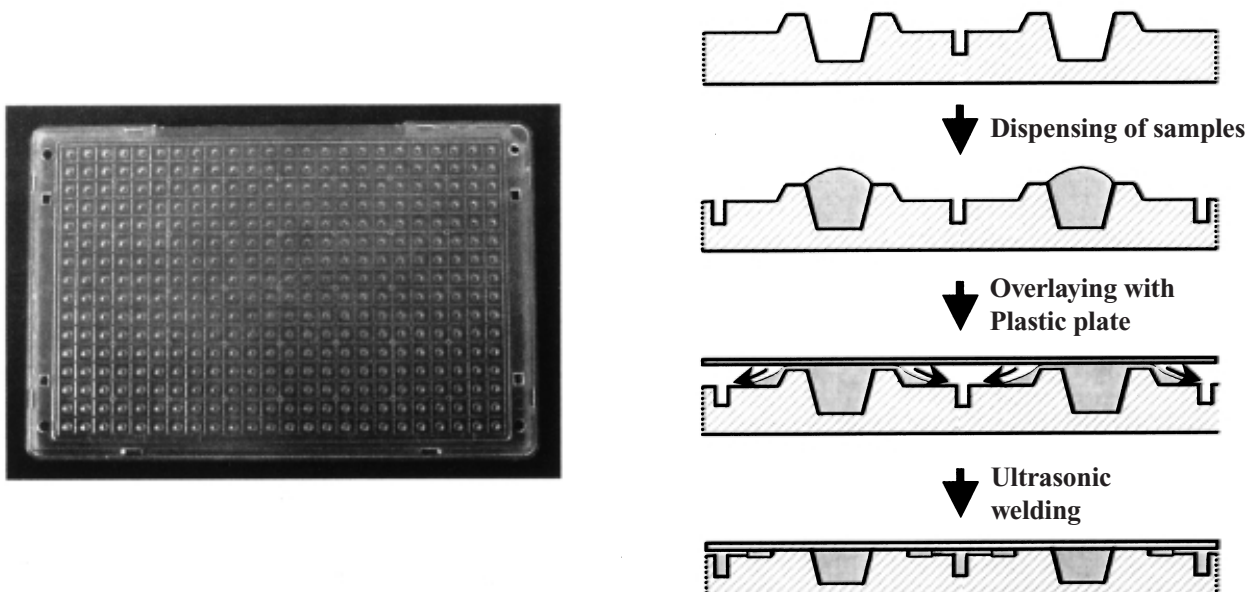

c)

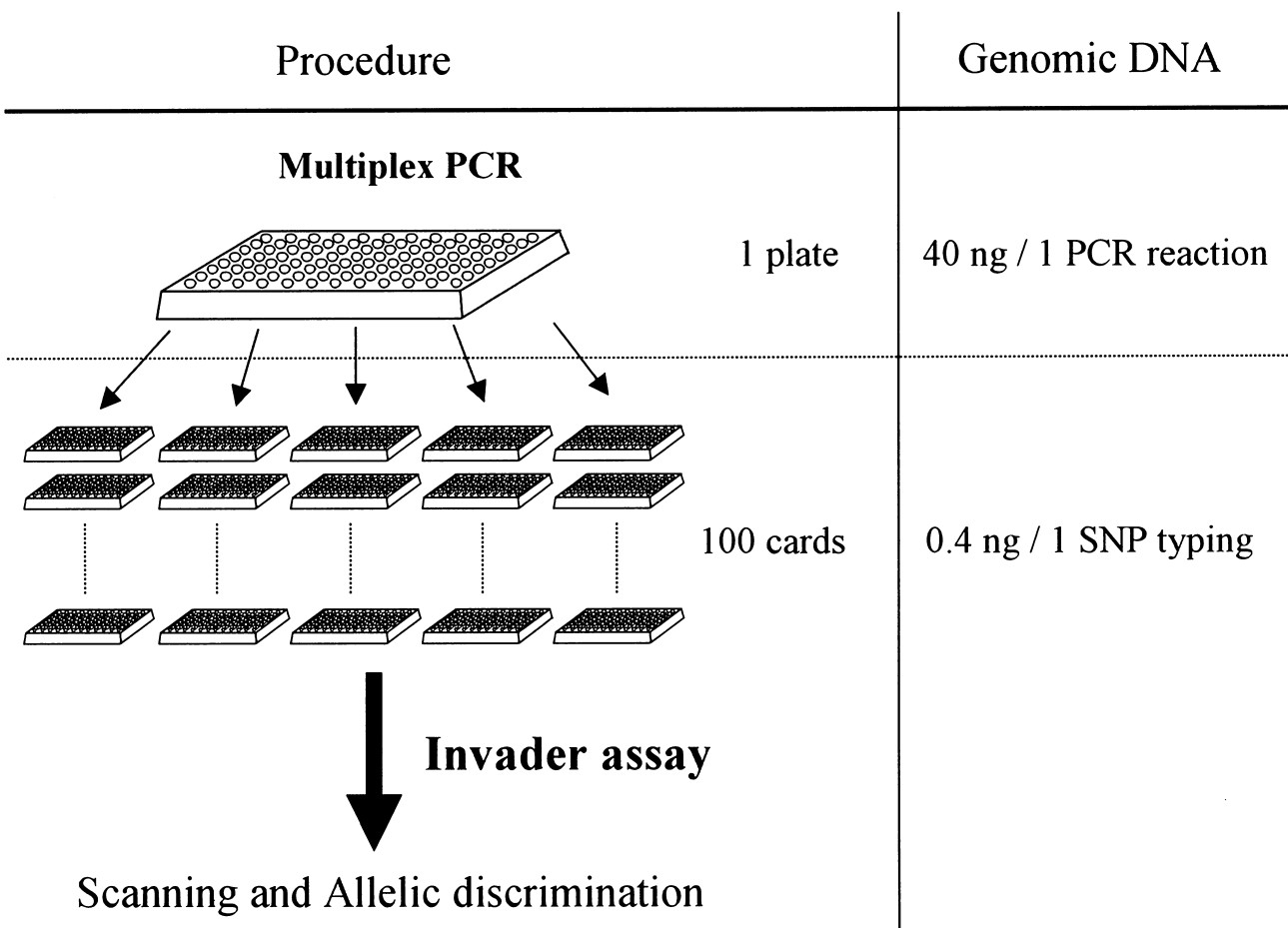

the source is limited and cannot be increased by simply spending more money. Although some promising new approaches have been reported, until now, none has successfully reduced to a practicable level the amount of genomic DNA required from an individual blood sample.

Here, however, we have reported a high-throughput and highly efficient tool for genotyping SNPs. Although it merely combines easily available methods, our modification provides a way to solve the most serious practical problems in genome-wide association studies. Using the automated sample-flow system, along with our newly developed 384well cards, we have established a protocol for genotyping up to 100 million SNPs per year.
Acknowledgments We thank Akemi Inose, Yoko Ariji, and Maki Takahashi for their technical assistance, and Kaori Sato for assistance in preparation of the manuscript. This work was supported a grant from the Japanese Millennium Project.

\section{References}

Collins FS, Guyer MS, Chakravarti A (1997) Variations on a thema: cataloging human DNA sequence variation. Science 278:1580-1581 Edwards MC, Gibbs RA (1994) Multiplex PCR: advantages, development and applications. PCR Methods Appl 3:S65-S75

Kellogg DE, Rybalkin I, Chen S, Mukhamedova N, Vlasik T, Siebert PD, Chenchik A (1994) TaqStart antibody: "hot start" PCR facili- 
tated by a neutralizing monoclonal antibody directed against Taq DNA polymerase. Biotechniques 16:1134-1137

Kruglyak L (1999) Prospects for whole-genome linkage disequilibrium mapping of common disease genes. Nat Genet 22:139-144

Lander ES, Schork NJ (1994) Genetic dissection of complex traits. Science 265:2037-2048

Lander ES (1996) The new genomics: global view of biology. Science 274:536-539

Lyamichev V, Mast AL, Hall JG, Prudent JR, Kaiser MW, Takova T, Kwiatkowski RW, Sander TJ, de Arruda M, Arco DA, Neri BP, Brow MA (1999) Polymorphism identification and quantitative detection of genomic DNA by invasive cleavage of oligonucleotide probes. Nat Biotechnol 17:292-296
Mein CA, Barratt BJ, Dunn MG, Siegmund T, Smith AN, Esposito L, Nutland S, Stevens HE, Wilson AJ, Phillips MS, Jarvis N, Law S, de Arruda M, Todd JA (2000) Evaluation of single nucleotide polymorphism typing with Invader on PCR amplicons and its automation. Genome Res 10:330-343

Risch N, Merikangas K (1996) The future of genetic studies of complex human diseases. Science 273:1516-1517

Ryan D, Nuccie B, Arvan D (1999) Non-PCR-dependent detection of the factor $\mathrm{V}$ Leiden mutation from genomic DNA using a homogeneous Invader microtiter plate assay. Mol Diagn 4:135-144 